\title{
MicroRNA-106b promotes the proliferation, migration and invasion of retinoblastoma cells by inhibiting the expression of $\mathrm{ZBTB} 4$ protein
}

\author{
WENJUAN BU $^{1 *}$, YANHUI WANG ${ }^{2 *}$ and XIANGRONG MIN ${ }^{1}$ \\ ${ }^{1}$ Department of Ophthalmology, Jining No. 1 People's Hospital, Jining, Shandong 272011; \\ ${ }^{2}$ Department of Fundus Surgery, Hebei Province Eye Hospital, Xingtai, Hebei 054000, P.R. China
}

Received January 20, 2017; Accepted December 6, 2017

DOI: $10.3892 /$ etm.2018.6811

\begin{abstract}
The present study investigated the function of microRNA (miR)-106b in the proliferation, migration and invasion of retinoblastoma $(\mathrm{RB})$ cells, and aimed to elucidate the underlying mechanism. A total of 56 patients with RB were enrolled in the present study. The expression of miR-106b in $\mathrm{RB}$ tissues was measured by reverse transcription quantitative polymerase chain reaction. After transfection with miR-106b mimics or miR-106b inhibitor, a Cell-Counting kit-8 assay was used to determine the proliferation of WERI-Rb-1 cells and a Transwell assay was employed to measure the migration and invasion of the cells. Western blot analysis was performed to determine the expression of zinc finger and BTB domain containing 4 (ZBTB4) protein. By silencing or overexpression of $\mathrm{ZBTB} 4$ protein, the biological functions of ZBTB4 in WERI-Rb-1 cells were studied. A dual luciferase reporter assay was performed to test whether ZBTB4 was a target gene of miR-106b. The expression of miR-106b in RB tissues was elevated and closely associated with the severity of the disease. Overexpression of miR-106b increased but inhibition of miR-106b expression decreased the proliferation, migration and invasion abilities of WERI-Rb-1 cells. In addition, overexpression of miR-106b decreased but inhibition of miR-106b expression increased ZBTB4 protein expression in WERI-Rb-1 cells. Similarly, overexpression of ZBTB4 reduced but inhibition of ZBTB4 expression promoted the proliferation, migration and invasion of WERI-Rb-1 cells Finally, miR-106b regulated the expression of ZBTB4 by binding to the 3 '-untranslated region of the ZBTB4 gene.
\end{abstract}

Correspondence to: $\mathrm{Dr}$ Xiangrong Min, Department of Ophthalmology, Jining No. 1 People's Hospital, 6 Jiankang Road, Jining, Shandong 272011, P.R. China

E-mail: lwgdf1@126.com

*Contributed equally

Key words: miR-106b, retinoblastoma, zinc finger and BTB domain containing 4
The present study demonstrated that increased expression of miR-106b in RB tissues is positively associated with the metastasis and differentiation of RB cells. As an oncogene, miR-106b promotes the proliferation, migration and invasion of WERI-Rb-1 cells by inhibiting the expression of ZBTB4 protein.

\section{Introduction}

Retinoblastoma (RB) is the most common type of primary intraocular malignant tumor in pediatric patients that has an occult onset and a high degree of malignancy (1). Without treatment, affected pediatric patients generally die within 1-2 years (2). Invasion and metastasis are the leading causes of death in pediatric patients with RB, making the disease a complex process that involves multiple genes, factors and procedures $(3,4)$. In recent years, enucleation has been excluded from the treatment options for RB, and different treatments are adopted according to the different stages of the tumor, aiming to retain the eyes and their functions (5). Chemoreduction, mainly systemic chemotherapy, has become the first-line approach for the clinical treatment of RB $(6,7)$. It greatly improved the survival rate of the patients without affecting the structure and function of eyeballs. However, failure of RB treatment remains common in clinical practice due to two reasons. First, tumor cells may develop resistance to drugs (8); furthermore, continued use of chemotherapeutic drugs may induce bone marrow suppression and secondary leukemia, increasing the mortality risk of the patients (9). Therefore, it is of great clinical significance to develop a safer and more effective adjuvant therapy for the treatment of patients with RB.

MicroRNAs (miRNAs or miRs) are non-coding small RNA molecules of 18-22 nucleotides in length that regulate mRNA translation by binding with the 3'-untranslated region (UTR) of their target mRNAs $(10,11)$. miRNA molecules widely exist in eukaryotes and participate in multiple pathological processes. It has been reported that a variety of miRNA molecules act as oncogenes or tumor-suppressor genes in RB (12). For instance, miR-21 promotes the occurrence and development of RB by activating phosphatase and tensin homologue (PTEN)/phosphoinositide-3 kinase (PI3K)/AKT 
signaling pathway, and miR-21 inhibitor blocks the function of miR-21 (13). Bai et al (14) reported that miR-125b promotes the proliferation and inhibits the apoptosis of RB cells by targeting DNA damage-regulated autophagy modulator 2 gene expression. Zhang et al (15) reported that miR-125a-5p enhances the proliferation of RB cells by activating taffazin-epithelial growth factor receptor signaling pathway.

miR-106b is a member of the miR-106-25 family, which also includes miR-25 and miR-93 (16,17). The expression of miR-106b in gastric cancer tissues is significantly higher than that in adjacent normal tissue, and abnormal expression of miR-106b is observed in the peripheral blood of gastric cancer patients, suggesting that miR-106b has an important role in the occurrence and development of gastric cancer (18). Dai et al (19) discovered that miR-106b promotes the invasion and migration of esophageal squamous cancer cells in a Kazakh population by downregulating the expression of Smad7. Li et al (20) reported that miR-106b increases the proliferation, invasion and migration of breast cancer cells by regulating the expression of $\alpha$ - $(1,3)$-fucosyltransferase. These studies suggest that miR-106b has varied functions and mechanisms of action in different types of tumor. However, the expression and mechanism of action of miR-106b in RB has remained to be elucidated. The present study investigated the function of miR-106b in the proliferation, invasion and migration of RB cells, and aimed to elucidate the underlying mechanisms.

\section{Patients and methods}

Patients. A total of 56 patients (34 males and 22 females; age range, 2 months-11 years; mean age, $35.5 \pm 8.5$ months) who received treatment for RB at Jining First People's Hospital between December 2013 and December 2015 were enrolled in the present study. No patients received any chemotherapy or other treatments prior to sampling. Additionally, patients with chronic, genetic or autoimmune diseases were excluded from the present study. Among the patients, 36 had RB in one eye and 20 had RB in both eyes. According to the degrees of optic nerve invasion, the patients were divided into four groups according to tumor necrosis metastasis staging system: N0, RB did not invade optic nerves $(n=9)$; N1, RB invaded optic papilla, but did not exceed sieve plate $(n=6) ; N 2, R B$ passed through sieve plate, but did not invade the end of optic nerves $(n=25)$; and N3, RB cells were identified at the end of optic nerves $(n=16)(21)$. Depending on whether RB cells were arranged into a Flexner-Winterstein rose ring, RB tissues were stratified into a high-differentiation group ( $n=39$ cases) and a poor-differentiation group $(n=17)$. Furthermore, normal retinal tissues were obtained from the adjacent tissues of RB tissues from 20 randomly selected patients. All procedures were approved by the Ethics Committee of Jining No. 1 People's Hospital. Written informed consent was obtained from parents or guardians of all pediatric patients.

Cell line, culture and transfection. The RB cell line WERI-Rb-1 (Cell Bank of the Chinese Academy of Sciences, Shanghai, China) was cultured in RPMI-1640 medium supplemented with $10 \%$ fetal bovine serum (BD Biosciences, Franklin Lakes, NJ, USA), $100 \mathrm{IU} / \mathrm{ml}$ penicillin and $100 \mathrm{IU} / \mathrm{ml}$ streptomycin at $37^{\circ} \mathrm{C}$ in an atmosphere with $5 \% \mathrm{CO}_{2}$ and $70 \%$ humidity. The cells were passaged once every four days, and those in the logarithmic growth phase were collected for experiments. On the day before transfection, the cells $\left(3 \times 10^{5}\right)$ were seeded onto 24-well plates containing RPMI-1640 without fetal bovine serum or antibiotics. The cells were divided into negative control (NC) and miR-106b mimics groups. When reaching $70 \%$ confluency, $1.5 \mu \mathrm{l}$ miR-negative control (NC), miR-106b mimics or miR-106b inhibitor (20 pmol/ $\mu \mathrm{l}$; RiboBio, Guangzhou, China) and $1 \mu \mathrm{l}$ liposome (Lipofectamine 2000; Thermo Fisher Scientific, Inc. Waltham, MA, USA) were mixed with $50 \mu$ l OptiMem medium (Thermo Fisher Scientific, Inc.), respectively, in individual Eppendorf tubes. After standing still for $5 \mathrm{~min}$, the mixtures in the two Eppendorf tubes were mixed and kept at room temperature for $20 \mathrm{~min}$, followed by addition into each culture well. After $6 \mathrm{~h}$ of incubation, the medium was replaced with fresh RPMI-1640 medium supplemented with $10 \%$ fetal bovine serum, and the cells were cultured under normal conditions prior to use. For RNA interference or overexpression of the zinc finger and BTB domain containing 4 (ZBTB4) gene, WERI-Rb-1 cells were transfected with lentiviral vectors LV-puro-shR-ZBTB4 and LV-puro-ZBTB4, respectively (HanBio, Shanghai, China) with a multiplicity of infection of 20 . An empty vector was used as a negative control. After incubation for $72 \mathrm{~h}$, the cells were harvested for further use.

Reverse transcription-quantitative polymerase chain reaction $(R T-q P C R)$. RB and control tissues $(100 \mathrm{mg})$ were ground into powder in liquid nitrogen and mixed with $1 \mathrm{ml} \mathrm{TRIzol}$ (Thermo Fisher Scientific, Inc.) for lysis. Total RNA was then extracted using the phenol chloroform method. The purity of the RNA was determined via the absorbance at $260 \mathrm{vs} .280 \mathrm{~nm}$ using ultraviolet spectrophotometry (Nanodrop ND2000; Thermo Fisher Scientific, Inc.). The complementary (c)DNA was obtained by reverse transcription from $1 \mu \mathrm{g}$ RNA and stored at $-20^{\circ} \mathrm{C}$. Reverse transcription of mRNA was performed using the PrimeScript ${ }^{\mathrm{TM}}$ II First Strand cDNA Synthesis kit (Takara, Dalian, China), and reverse transcription of miRNA was performed using the One Step PrimeScript miRNA cDNA Synthesis kit (Takara).

The expression of miR-106b was determined using the SYBR PrimeScript RT-PCR Kit (Takara, Dalian, China), using U6 as an internal reference. The reaction system $(20 \mu \mathrm{l})$ contained $10 \mu \mathrm{l}$ real-time quantitative PCR mix, $0.5 \mu \mathrm{l}$ upstream primer (miR-106b; 5'-TAAAGTGCTGACAGTGCAGAT-3'), $0.5 \mu 1$ downstream universal primer (provided by the kit), $2 \mu \mathrm{l}$ cDNA and $7 \mu \mathrm{l}$ double-distilled (dd) $\mathrm{H}_{2} \mathrm{O}$. The reaction protocol was as follows: Initial denaturation at $95^{\circ} \mathrm{C}$ for $10 \mathrm{~min}$, followed by 40 cycles of $95^{\circ} \mathrm{C}$ for $1 \mathrm{~min}$ and $60^{\circ} \mathrm{C}$ for $30 \mathrm{sec}$ (iQ5; Bio-Rad Laboratories, Inc., Hercules, CA, USA). The $2^{-\Delta \Delta \mathrm{Cq}}$ method (22) was used to calculate the relative expression of miR-106b against the internal reference. Each sample was tested in triplicate for PCR amplification.

The SYBR Green RT-qPCR kit (Kapa Biosystems, Wilmington, MA, USA) was used to detect mRNA expression of ZBTB4, using GAPDH as an internal reference. The reaction system $(20 \mu \mathrm{l})$ was composed of $10 \mu \mathrm{l}$ SYBR EX Taq-Mix, $0.5 \mu 1$ upstream primer (ZBTB4, 5'-TCCCTTTTGCACTGA GGCTT-3'; GAPDH, 5'-CGGAGTCAACGGATTTGGTCG 
TAT-3'), $0.5 \mu 1$ downstream primer (ZBTB4, 5'-AGAAGG GACTTGAAGCAGCC-3'; GAPDH, 5'-AGCCTTCTCCAT GGTGGTGAAGAC-3'), $5 \mu 1 \mathrm{cDNA}$ and $4 \mu 1 \mathrm{dd}_{2} \mathrm{O}$. The PCR conditions were as follows: Initial denaturation at $95^{\circ} \mathrm{C}$ for $10 \mathrm{~min}$; 40 cycles of denaturation at $95^{\circ} \mathrm{C}$ for $1 \mathrm{~min}$, annealing at $60^{\circ} \mathrm{C}$ for $40 \mathrm{sec}$ and elongation at $72^{\circ} \mathrm{C}$ for $30 \mathrm{sec}$; and final elongation at $72^{\circ} \mathrm{C}$ for $1 \mathrm{~min}$ (iQ5; Bio-Rad Laboratories, Inc.). The $2^{-\Delta \Delta C q}$ method was used to calculate the relative expression of ZBTB4 mRNA against GAPDH. Each sample was tested in triplicate.

Cell Counting kit $8(C C K-8)$ assay. Cells were seeded at $2,000 /$ well in 96 -well plates for transfection. At $48 \mathrm{~h}$ after transfection, the cells were subjected to the CCK- 8 assay for the detection of proliferation. At 0,24 and $48 \mathrm{~h}$, the medium was discarded and the cells were washed with PBS twice, followed by addition of $20 \mu \mathrm{l}$ CCK- 8 reaction reagent $(5 \mathrm{~g} / \mathrm{l})$ diluted in medium. On the last day, $150 \mu \mathrm{l}$ CCK- 8 reaction solution was added and the cells were incubated at $37^{\circ} \mathrm{C}$ for $2 \mathrm{~h}$. The absorbance of each well was measured at $490 \mathrm{~nm}$ for plotting the cell proliferation curves. Each group was tested in 3 replicate wells and the values were averaged. Each experiment was performed in triplicate.

Transwell assay. For the invasion assay, Matrigel was thawed at $4^{\circ} \mathrm{C}$ overnight and diluted at 1:2 with serum-free RPMI-1640 medium. The mixture (50 $\mu \mathrm{l})$ was evenly smeared into the upper chambers of a Transwell insert (pore size, $0.8 \mu \mathrm{m}$; EMD Millipore, Billerica, MA, USA) and incubated at $37^{\circ} \mathrm{C}$ for $1 \mathrm{~h}$ to solidify. For both the invasion and migration assay, $1 \times 10^{5}$ cells from each group were then seeded into the upper chamber in $200 \mu 1$ serum-free RPMI-1640 medium. In addition, $500 \mu \mathrm{l}$ RPMI-1640 medium supplemented with $10 \%$ fetal bovine serum was added into the lower chamber. After $24 \mathrm{~h}$, the cells in the upper chambers were wiped off. After fixing of the membranes with $4 \%$ formaldehyde for $10 \mathrm{~min}$, the membrane was stained at room temperature for 2 min using the Giemsa method for microscopic observation of 5 random fields (magnification, x200). The number of cells that had transgressed through the membrane was calculated for the evaluation of cell invasion and migration ability. All procedures were performed on ice with pipetting tips being cooled at $4^{\circ} \mathrm{C}$.

Western blot analysis. Tissues or cells were ground into powder in liquid nitrogen and $100 \mathrm{mg}$ of the powder was mixed with $100 \mu 1$ pre-cooled radioimmunoprecipitation assay lysis buffer containing 1\% phenylmethylsulfonyl fluoride for lysis overnight at $4^{\circ} \mathrm{C}$. The mixture was then centrifuged at $12,000 \times \mathrm{g}$ and $4^{\circ} \mathrm{C}$ for $10 \mathrm{~min}$. The supernatant was used to determine the protein concentration by the bicinchoninic acid protein concentration determination kit (cat. no. RTP7102; Real-Times Biotechnology Co., Ltd., Beijing, China). Protein samples $(50 \mu \mathrm{g})$ were then mixed with 5X SDS loading buffer prior to denaturation in a boiling water bath for $10 \mathrm{~min}$. Subsequently, the samples $(10 \mu \mathrm{l})$ were subjected to $10 \%$ SDS-PAGE at $100 \mathrm{~V}$. The resolved proteins were transferred to polyvinylidene difluoride membranes (Bio-Rad Laboratories, Inc.) on ice $(250 \mathrm{~mA}, 1 \mathrm{~h})$ and blocked with $50 \mathrm{~g} / 1$ skimmed milk at room temperature for $1 \mathrm{~h}$. The membranes were then incubated with rabbit anti-human ZBTB4 (1:1,000 dilution; cat. no. ab106554; Abcam, Cambridge, UK) and GAPDH (1:4,000 dilution; cat. no. ab9485; Abcam) polyclonal primary antibodies at $4^{\circ} \mathrm{C}$ overnight. After extensive washing with PBS containing Tween-20 (PBST) for 5 times for 5 min each, the membranes were incubated with polyclonal goat anti-rabbit horseradish peroxidase-conjugated secondary antibody (1:4,000 dilution; cat. no. ab6721; Abcam) for $1 \mathrm{~h}$ at room temperature prior to washing with PBST as above. The membrane was then developed with an enhanced chemiluminescence detection kit (Sigma-Aldrich; Merck KGaA, Darmstadt, Germany) for imaging. Image lab v3.0 software (Bio-Rad Laboratories, Inc.) was used to acquire and analyze imaging signals. The relative expression of ZBTB4 protein was calculated against GAPDH.

Dual luciferase reporter assay. Bioinformatics prediction is a powerful tool for the study of the functions of miRNAs. To understand the regulatory mechanisms of miR-106b in RB, miRanda (http://www.microrna.org/microrna/home.do), TargetScan (http://www.targetscan.org), PiTa (http://genie. weizmann.ac.il/pubs/mir07/mir07_data.html), RNAhybrid (http://bibiserv.techfak.uni-bielefeld.de/rnahybrid/) and PICTAR (http://pictar.mdc-berlin.de/) were used to predict mRNAs that could be targeted by miR-106b, and identified that miR-106b was able to potentially regulate ZBTB4. According to the results of the bioinformatics analysis, wild-type (WT) and mutant seed regions of miR-106b in the 3'-UTR of the ZBTB4 gene were chemically synthesized, Spe-1 and HindIII restriction sites were added, and the sequences were cloned into pMIR-REPORT luciferase reporter plasmids (Ambion; Thermo Fisher Scientific, Inc.). Plasmids $(0.5 \mu \mathrm{g})$ with WT or mutant 3'-UTR DNA sequences were co-transfected with miR-106b mimics (100 nM; Sangon Biotech) into 293T cells. After cultivation for $24 \mathrm{~h}$, the cells were lysed using a dual luciferase reporter assay kit (Promega Corp., Madison, WI, USA) according to the manufacturer's protocols, and the fluorescence intensity was measured usinga GloMax 20/20 luminometer (Promega Corp.). Using Renilla fluorescence activity as an internal reference, the fluorescence values of each group of cells were measured.

Statistical analysis. Statistical analysis was performed using SPSS 17.0 software (IBM Corp., Armonk, NY, USA). Measurement data were expressed as the mean \pm standard deviation. Differences between two groups were compared using Student's t-test. Multigroup comparisons were made using one-way analysis of variance. In the case of homogeneity of variance, the Least Significant Difference and the Student-Newman-Keuls methods were used; in the case of heterogeneity of variance, Tamhane's T2 or Dunnett's T3 method was used. $\mathrm{P}<0.05$ was considered to indicate a statistically significant difference.

\section{Results}

Expression of miR-106b in RB tissues is elevated and closely associated with the disease severity. To measure 
A

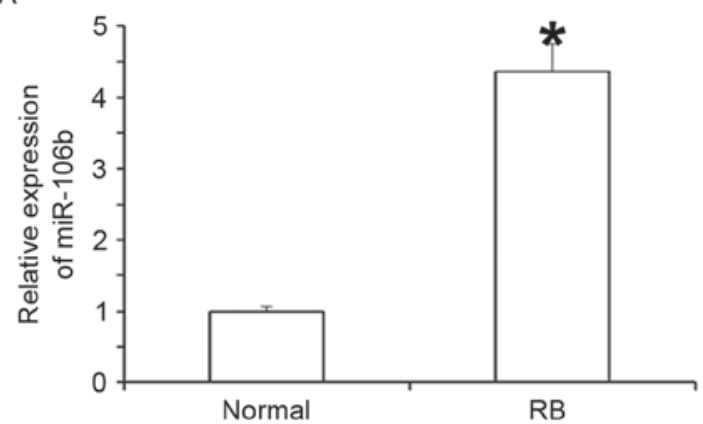

C

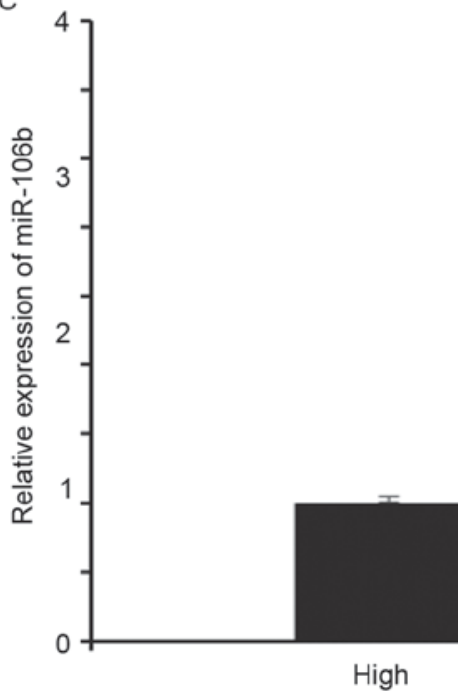

B

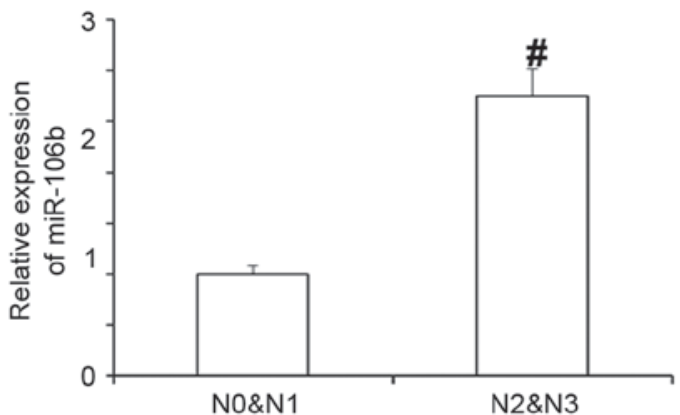

Figure 1. Expression of miR-106a in (A) RB tissues and normal tissues, (B) patients with different degrees of optic nerve invasion and (C) RB tissues of different degrees of differentiation. Relative expression of miR-106b was measured using reverse transcription-quantitative polymerase chain reaction. AJCC 2017 grading system for optic nerve invasion: N0, RB did not invade optic nerves ( $n=9)$; N1, RB invaded optic papilla, but did not exceed sieve plate ( $n=6$ ); $\mathrm{N} 2$, RB passed through sieve plate, but did not invade the end of optic nerves $(n=25)$; and N3, RB cells were identified at the end of optic nerves ( $n=16)$. ${ }^{*} \mathrm{P}<0.05$ vs. normal tissues; ${ }^{*} \mathrm{P}<0.05$ vs. $\mathrm{N} 0$ and $\mathrm{N} 1 ;{ }^{*} \mathrm{P}<0.05$ vs. high-differentiation group. RB, retinoblastoma; miR, microRNA.

the expression of miR-106b in RB tissues, RT-qPCR was performed. The results indicated that miR-106b levels in RB tissues were significantly higher than those in normal tissues $(\mathrm{P}<0.05$; Fig. 1A). In addition, the levels of miR-106b in the $\mathrm{N} 2$ and $\mathrm{N} 3$ groups were significantly higher than those in the N0 and N1 groups $(\mathrm{P}<0.05$; Fig. 1B). Furthermore, miR-106b expression in the poor-differentiation group was significantly higher than that in the high-differentiation group $(\mathrm{P}<0.05$; Fig. 1C). These results suggest that the expression of miR-106b in RB tissues is elevated and closely associated with the severity of the disease.

Overexpression of miR-106b increases but inhibition of miR-106b expression decreases the proliferation ability of $W E R I-R b-1$ cells. To determine the proliferation of WERI-Rb-1 cells, a CCK-8 assay was used. The results indicated that the absorbance of cells transfected with miR-106b mimics was significantly higher than that in the miR-NC group at 24 and $48 \mathrm{~h}(\mathrm{P}<0.05)$, while the absorbance of cells transfected with miR-106b inhibitor was significantly lower than that in the miR-NC group at 24 and $48 \mathrm{~h}(\mathrm{P}<0.05$; Fig. 2). This result indicated that overexpression of miR-106b increases but inhibition of miR-106b expression decreases the proliferation ability of WERI-Rb-1 cells.

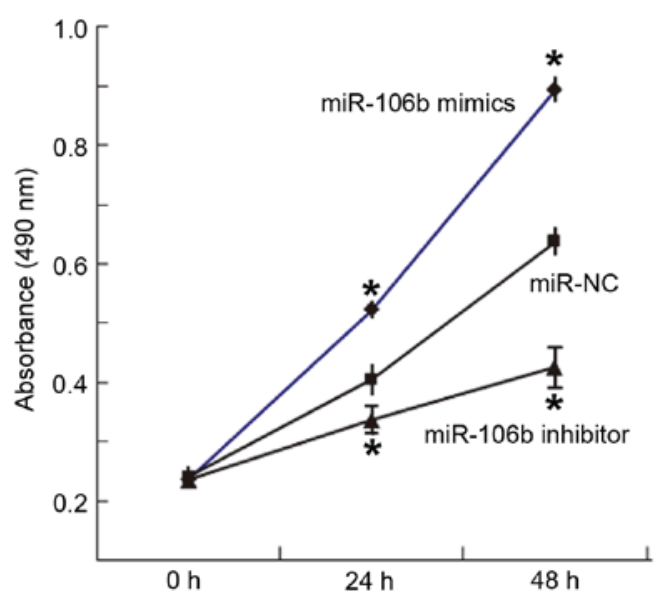

Figure 2. Proliferation of WERI-Rb-1 cells at 0, 24 and $48 \mathrm{~h}$ after transfection with miR-NC, miR-106b mimics or miR-106b inhibitor. A Cell Counting kit- 8 assay was used to determine the proliferation of the cells. The absorbance of each well was measured at $490 \mathrm{~nm}$ with a microplate reader and cell proliferation curves were plotted. ${ }^{*} \mathrm{P}<0.05$ vs. miR-NC group at the respective time-point. miR, microRNA; NC, negative control.

Overexpression of miR-106b increases but inhibition of miR-106b expression decreases the migration and invasion abilities of WERI-Rb-1 cells. To study the effect of 
A

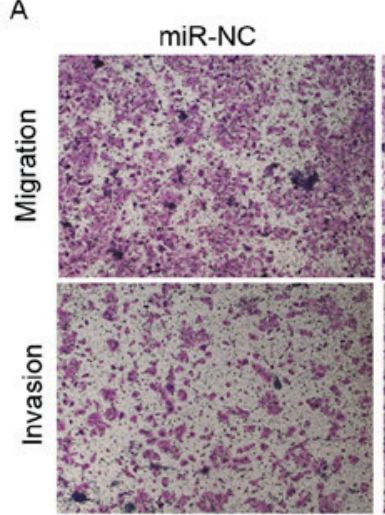

C

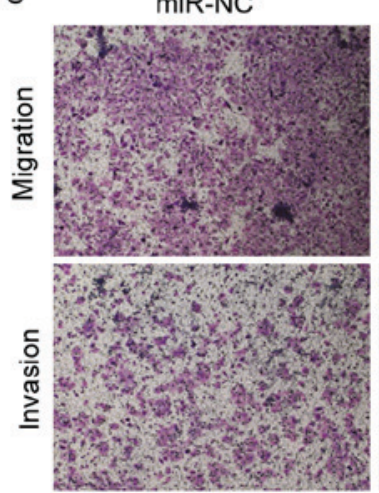

miR-106b mimics

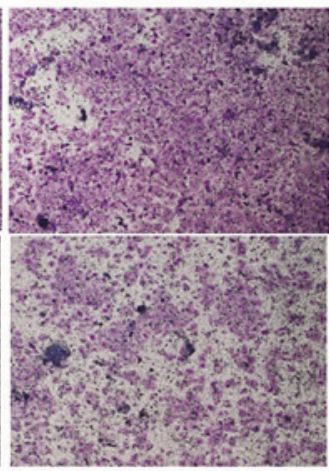

miR-106b inhibitor

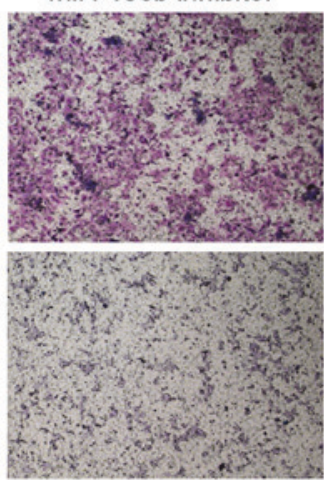

B

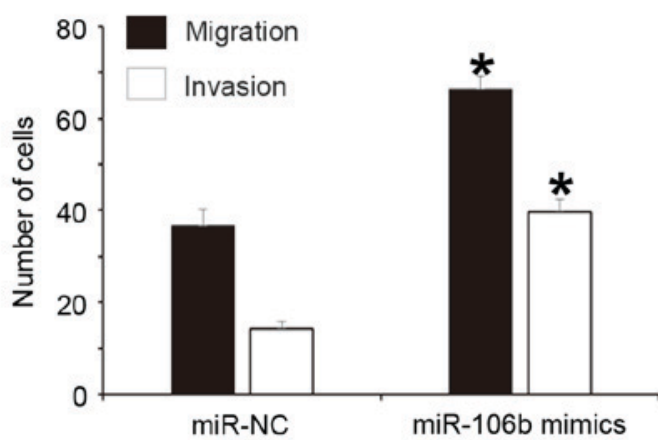

D

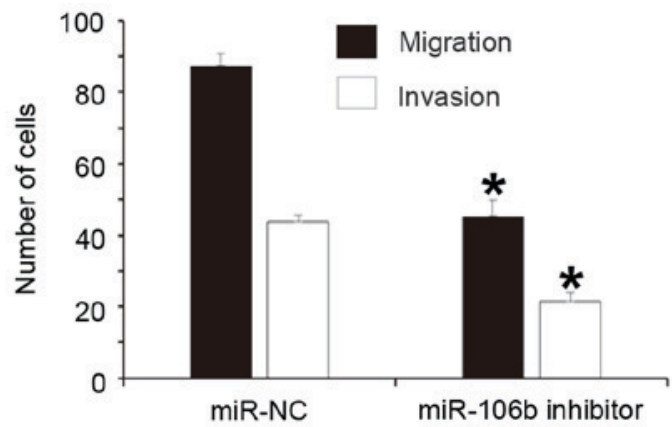

Figure 3. Effect of miR-106b on the migration and invasion abilities of WERI-Rb-1 cells. (A) Representative mages and (B) numbers of migrated and invaded cells after transfection with miR-NC or miR-106b mimics. (C) Representative mages and (D) numbers of migrated and invaded cells after transfection with miR-NC or miR-106b inhibitor. Magnification, x100. " $\mathrm{P}<0.05$ vs. miR-NC group. miR, microRNA; NC, negative control.

miR-106b on the migration and invasion of WERI-Rb-1 cells, a Transwell assay was performed. The results indicated that the numbers of cells in the miR-106b mimics group that transgressed through the Transwell membrane in the migration and invasion assays were significantly higher than those in the miR-NC group $(\mathrm{P}<0.05$ for both; Fig. 3A and $\mathrm{B})$. In addition, the numbers of cells in the miR-106b inhibitor group that transgressed through the Transwell membrane in the migration and invasion assays were significantly reduced compared with those in the miR-NC group $(\mathrm{P}<0.05$ for both; Fig. 3C and D). These results suggest that overexpression of miR-106b increases but inhibition of miR-106b expression decreases the migration and invasion abilities of WERI-Rb-1 cells.

Overexpression of miR-106b decreases but inhibition of miR-106b expression increases ZBTB4 protein expression in WERI-Rb-1 cells. To examine the expression of ZBTB4 protein, western blot analysis was employed. The results indicated that ZBTB4 expression in cells transfected with miR-106b mimics was significantly reduced compared with that in the respective miR-NC group $(\mathrm{P}<0.05)$, but that in cells transfected with miR-106b inhibitor was significantly increased $(\mathrm{P}<0.05$; Fig. 4A). In addition, WERI-Rb-1 cells with silencing of ZBTB4 by transfection with lentiviral vector had a significantly reduced expression of ZBTB4 protein compared with that in the respective miR-NC group $(\mathrm{P}<0.05)$, while cells with overexpression of ZBTB4 by transfection with lentiviral vector had a significantly enhanced expression of ZBTB4 protein compared with that in the NC group $(\mathrm{P}<0.05$; Fig. 4B). The results indicate that overexpression of miR-106b decreases but inhibition of miR-106b expression increases ZBTB4 protein expression in WERI-Rb-1 cells.

Overexpression of $Z B T B 4$ reduces but inhibition of $Z B T B 4$ expression promotes the proliferation, migration and invasion of WERI-Rb-1 cells. To assess the effect of ZBTB4 protein expression on the biological function of WERI-Rb-1 cells, a CCK-8 assay and Transwell assay were performed on cells with silenced expression or overexpression of ZBTB4. The results indicated that the absorbance of cells transfected with siR-ZBTB4 was significantly higher than that of cells transfected with siR-NC at 24 and $48 \mathrm{~h}(\mathrm{P}<0.05)$, while the absorbance of cells in the ZBTB4 group was significantly lower than that of cells transfected with $\mathrm{NC}$ at 24 and $48 \mathrm{~h}$ (Fig. 5). The Transwell assay indicated that the numbers of cells in the siR-ZBTB4 group that transgressed through the Transwell membrane in the migration and invasion assays were significantly higher than those in the siR-NC group $(\mathrm{P}<0.05$ for both; Fig. 6A and $\mathrm{B})$. In addition, the numbers of cells in the ZBTB4 group that transgressed through the Transwell membrane in the migration and invasion assays were significantly reduced compared with those in the NC group $(\mathrm{P}<0.05$ for both; Fig. $6 \mathrm{C}$ and $\mathrm{D})$. These results suggest that overexpression of ZBTB4 reduces but inhibition of ZBTB4 expression promotes the proliferation, migration and invasion of WERI-Rb-1 cells. 

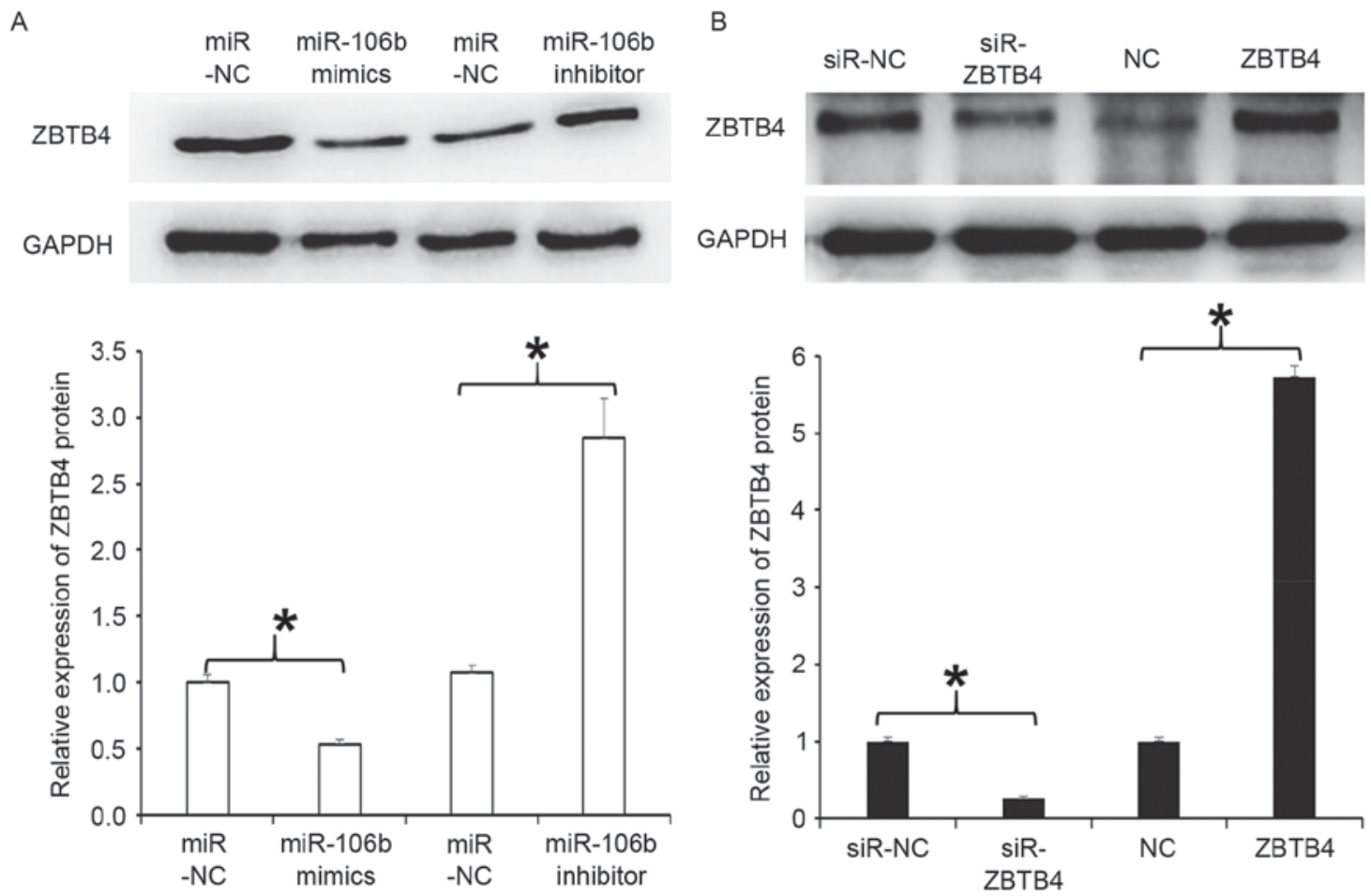

Figure 4. Effect of miR-106b on ZBTB4 protein expression in WERI-Rb-1 cells. (A) Expression of ZBTB4 protein in cells transfected with miR-106b mimics or inhibitor. (B) Expression of ZBTB4 protein in cells transfected with siR-ZBTB4 or ZBTB4. Western blot analysis was used to measure ZBTB4 protein expression. " $\mathrm{P}<0.05$. miR, microRNA; NC, negative control; siR, small interfering RNA; ZBTB4, zinc finger and BTB domain containing 4.

miR-106b inhibits the expression of ZBTB4 by binding with the 3'-UTR of ZBTB4 $m R N A$. To understand whether miR-106b directly targets ZBTB4, a dual luciferase reporter assay was performed. The results demonstrated that transfection with miR-106b mimics and pMIR-REPORT-wild-type ZBTB4 led to significantly reduced fluorescence intensity compared with that in the negative control miR-transfected sample $(\mathrm{P}<0.05)$, while transfection with miR-106b mimics and pMIR-REPORT-mutant ZBTB4 resulted in similar fluorescence intensity compared with that in the negative control miR-transfected sample ( $P>0.05$; Fig. 7). This result indicates that miR-106b regulates the expression of ZBTB4 by binding with the 3'-UTR of ZBTB4 mRNA.

\section{Discussion}

Certain miRNAs have important roles in the occurrence and development of tumors. Members of the miR-106b-25 family display abnormal expression in multiple tumor tissues and peripheral blood, participating in the regulation of proliferation, apoptosis, invasion and metastasis of tumors (23). For instance, the expression of the miR-106b-25 cluster is elevated in recurrent acute myeloid leukemia in pediatric patients caused by mixed lineage leukemia gene rearrangement, suggesting that miR-106b-25 is closely associated with the recurrence of acute myeloid leukemia (24). Zheng et al (25) discovered that miR-106b induces radiotherapy resistance of colon cancer cells by regulating the PTEN/PI3K/AKT signaling pathway and p21 expression. It has been reported that miR-106b-25 cluster expression is elevated in gastric cancer tissues and peripheral blood of patients, and promotes the proliferation,

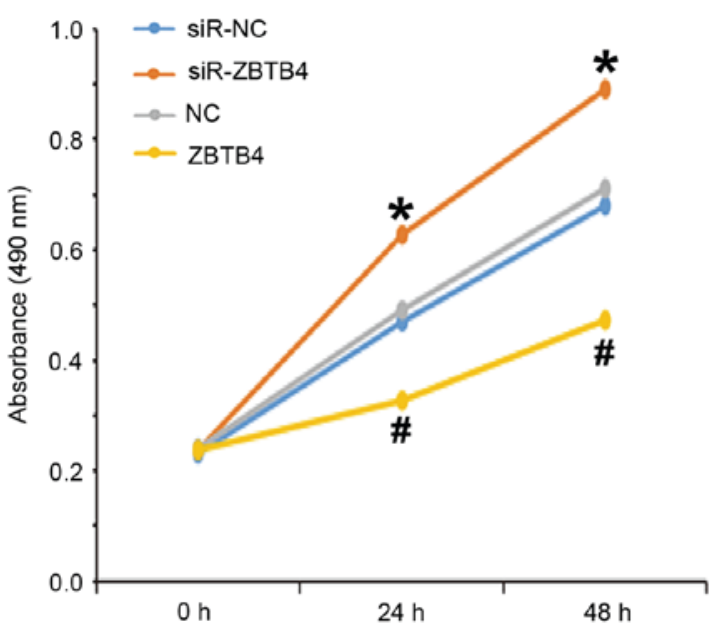

Figure 5. Effect of ZBTB4 expression on the proliferation of WERI-Rb-1 cells. Proliferation of WERI-Rb-1 cells with silencing or overexpression of ZBTB4 at 0,24 and 48 h. ${ }^{*} \mathrm{P}<0.05$ vs. siR-NC group; ${ }^{~} \mathrm{P}<0.05$ vs. NC group. $\mathrm{NC}$, negative control; siR, small interfering RNA; ZBTB4, zinc finger and BTB domain containing 4 .

invasion and metastasis of gastric cancer cells (23). In the present study, it was discovered that miR-106b expression is significantly increased in RB tissues, and patients at $\mathrm{N} 2$ and N3 stages had higher miR-106b levels than patients at N0 and N1 stages, suggesting that miR-106b expression is associated with the invasion and metastasis of RB. Regarding the degree of differentiation, miR-106b expression in the poor-differentiation group was significantly higher than that in the high-differentiation group, indicating that miR-106b expression is associated with RB cell differentiation. At the 
A
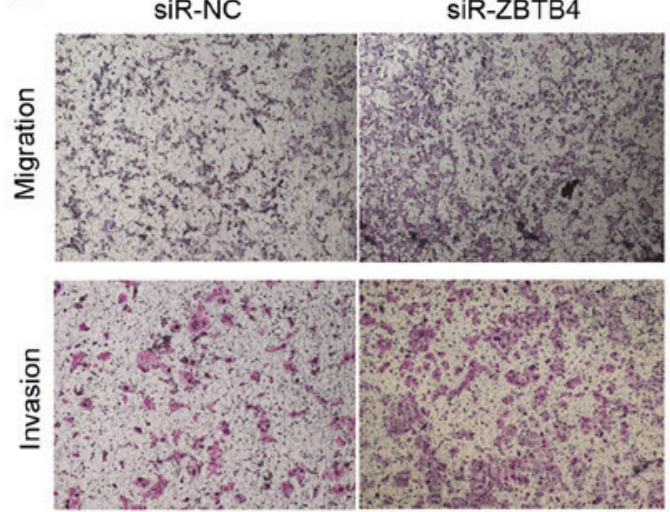

C
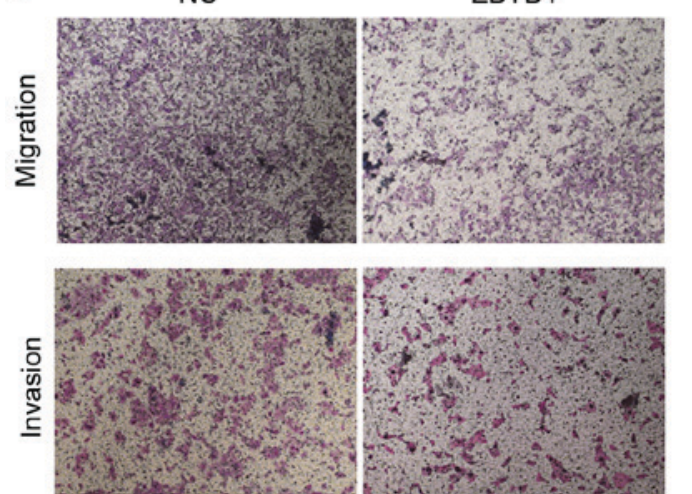

B

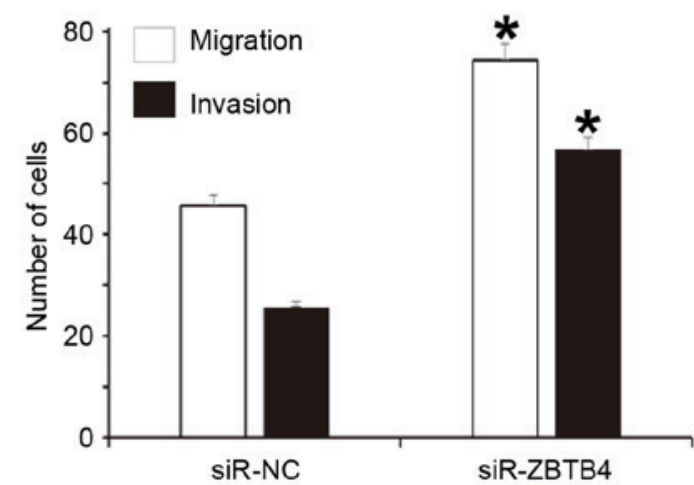

D

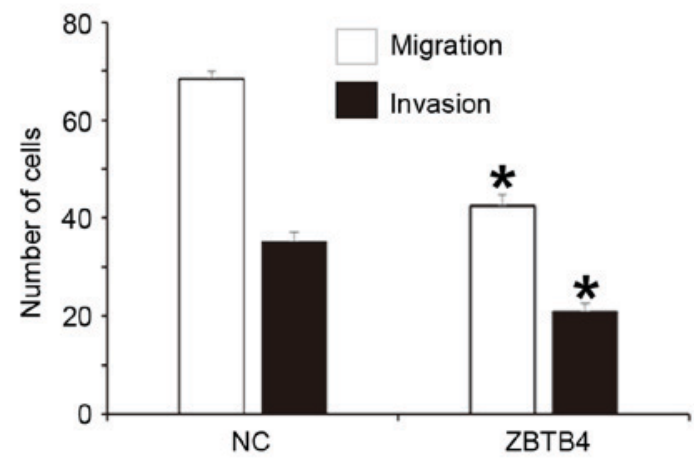

Figure 6. Effect of ZBTB4 expression on the migration and invasion abilities of WERI-Rb-1 cells. (A) Representative images and (B) numbers of migrated and invaded cells after transfection with siR-NC or siR-ZBTB4. " $\mathrm{P}<0.05$ vs. siR-NC group. (C) Representative mages and (D) numbers of migrated and invaded cells after transfection with NC or ZBTB4. Magnification, $\mathrm{x} 100$. " $\mathrm{P}<0.05$ vs. NC group. NC, negative control; siR, small interfering RNA; ZBTB4, zinc finger and BTB domain containing 4 .

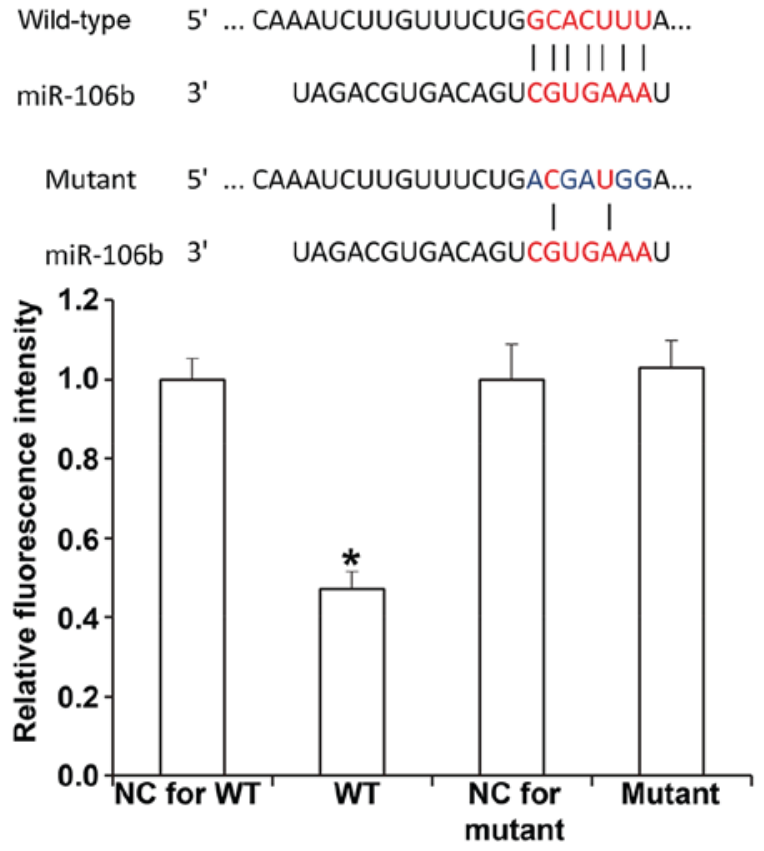

Figure 7. Fluorescence values of $293 \mathrm{~T}$ cells that were co-transfected with miR-106b negative control and wild-type 3'-untranslated DNA sequences of ZBTB4 (NC for WT group), miR-106b mimics and wild-type ZBTB4 (WT group), miR-106b NC and mutant ZBTB4 (NC for mutant group), or miR-106b mimics and mutant 3'-untranslated DNA sequences of ZBTB4 (Mutant group). A dual luciferase reporter assay was used to evaluate the interaction between miR-106b and ZBTB4. ${ }^{*} \mathrm{P}<0.05$ vs. negative control. ZBTB4, zinc finger and BTB domain containing 4; miR, microRNA. cellular level, silencing of miR-106b expression inhibited the proliferation, migration and invasion of WERI-Rb-1 cells, while overexpression of miR-106b had the opposite effect, suggesting that miR-106b acts as an oncogene in RB.

The ZBTB protein family is a class of proteins that have a BTB domain at the $\mathrm{N}$-terminus and multiple zinc finger domains at the C-terminus (26,27). Most members of the ZBTB family have a transcription factor function. ZBTB4, a member of the ZBTB family comprising 1,013 amino acids, has $1 \mathrm{BTB}$ domain at $\mathrm{N}$-terminus and $6 \mathrm{C}_{2} \mathrm{H}_{2}$-type zinc finger structures at the $\mathrm{C}$-terminus. ZBTB4 is a phosphorylation substrate for homeodomain-interacting protein kinase 2, and its phosphorylation induces the degradation of ZBTB4 protein and DNA injury (28). It was also reported that ZBTB4 directly binds to the promoter of p21 with the assistance of ZBTB17, inhibits p21 transcription, prevents cell cycle arrest via p21 after DNA injury and induces apoptosis (29). The bioinformatics study performed in the present study suggested that ZBTB4 mRNA is one of the potential targets of miR-106b. Western blot analysis and the dual luciferase reporter assay demonstrated that miR-106b directly binds to the 3'-UTR of ZBTB4 and reduces the expression of ZBTB4 protein. By contrast, miR-106b inhibitor increased the expression of ZBTB4 protein in WERI-Rb-1 cells, suggesting that ZBTB4 is a target gene of miR-106b. Further loss- and gain-of-function experiments indicated that overexpression of ZBTB4 inhibits the proliferation, migration and invasion of WERI-Rb-1 cells, 
while silencing of ZBTB4 expression reduces the proliferation, migration and invasion of WERI-Rb-1 cells, indicating that ZBTB4 has a tumour suppressor function in WERI-Rb-1 cells. At present, there are few reports on the effect of miR-106b in RB. Similar with breast cancer and cerebral tumors $(30,31)$, miR-106b promotes the occurrence and development of RB. More importantly, the present study demonstrated that miR-106b regulates the biological activities of RB via ZBTB4 gene and elucidates its underlying mechanism. In conclusion, miR-106b promotes the proliferation, migration and invasion of RB cells by inhibiting ZBTB4 expression. It is a potential therapeutic target and biomarker for RB.

\section{Acknowledgements}

The authors would like to thank Jining No. 1 People's Hospital (Jining, China) for the provision of facilities. The authors would also like to thank Dr Shuyin Sun and Dr Jie Feng of the Department of Ophthalmology, Jining No. 1 People's Hospital for reading the manuscript and providing valuable suggestions.

\section{Funding}

No funding was received.

\section{Availability of data and materials}

The analyzed data sets generated during the present study are available from the corresponding author on reasonable request.

\section{Authors' contributions}

WB, YW and XM designed the study. WB and YW performed the experiments, and $\mathrm{WB}, \mathrm{YW}$ and $\mathrm{XM}$ analyzed the data. All authors interpreted the results, and produced and approved the final manuscript.

\section{Ethics approval and consent to participate}

All procedures were approved by the Ethics Committee of Jining No. 1 People's Hospital. Written informed consent was obtained from parents or guardians of all pediatric patients.

\section{Patient consent for publication}

Not applicable.

\section{Competing interests}

The authors declare that they have no competing interests.

\section{References}

1. Soliman SE, Dimaras H, Khetan V, Gardiner JA, Chan HS, Héon E and Gallie BL: Prenatal versus postnatal screening for familial retinoblastoma. Ophthalmology 123: 2610-2617, 2016.

2. Natalino RJ, Antoneli CB, Ribeiro KC, Campos AH and Soares FA: Immunohistochemistry of apoptosis-related proteins in retinoblastoma. Pathol Res Pract 212: 1144-1150, 2016.

3. Gupta AK, Jones M, Prelog K, Bui J, Zhu J, Ng A and Dalla-Pozza L: Pineal cysts-A benign association with familial retinoblastoma. Pediatr Hematol Oncol 33: 408-414, 2016.
4. Tian T, Ji XD, Zhang Q, Peng J and Zhao PQ: A delayed diagnosis of unsuspected retinoblastoma in an in vitro fertilisation infant with retinopathy of prematurity. Int J Ophthalmol 9: 1361-1363, 2016.

5. Silva BB, Sapienza L, Castro DG, Ferreira DDV, Leão CR, Neves DFGDS, Silva B, Aiza A, Scintini AC, Pellizzon C and Regalin M: Eye plaque brachytherapy for retinoblastoma-A uni-institutional retrospective analysis of 40 eyes in 38 patients treated from 2001 to 2014. Int J Radiat Oncol Biol Phys 96: E554, 2016.

6. Assayag F, Nicolas A, Vacher S, Dehainault C, Bieche I, Meseure D, Aerts I, Cassoux N, Houdayer C, Doz F and Decaudin D: Combination of carboplatin and bevacizumab is an efficient therapeutic approach in retinoblastoma patientderived xenografts. Invest Ophthalmol Vis Sci 57: 4916-4926, 2016.

7. Shehata HH, Abou Ghalia AH, Elsayed EK, Ahmed Said AM and Mahmoud SS: Clinical significance of high levels of survivin and transforming growth factor beta-1 proteins in aqueous humor and serum of retinoblastoma patients. J AAPOS 20: 444. e441-444.e449, 2016.

8. Singh U, Malik MA, Goswami S, Shukla S and Kaur J: Epigenetic regulation of human retinoblastoma. Tumour Biol 37: 14427-14441, 2016.

9. Malorni L, Piazza S, Ciani Y, Guarducci C, Bonechi M, Biagioni C, Hart CD, Verardo R, Di Leo A and Migliaccio I: A gene expression signature of Retinoblastoma loss-of-function is a predictive biomarker of resistance to palbociclib in breast cancer cell lines and is prognostic in patients with ER positive early breast cancer. Oncotarget 7: 68012-68022, 2016.

10. Jin D and Lee H: Prioritizing cancer-related microRNAs by integrating microRNA and mRNA datasets. Sci Rep 6: 35350 , 2016.

11. Lima TI, Araujo HN, Menezes ES, Sponton CH, Araújo MB, Bomfim LH, Queiroz AL, Passos MA, E Sousa TA, Hirabara SM, et al: Role of microRNAs on the regulation of mitochondrial biogenesis and insulin signaling in skeletal muscle. J Cell Physiol 232: 958-966, 2017.

12. de Carvalho IN, de Freitas RM and Vargas FR: Translating microRNAs into biomarkers: What is new for pediatric cancer? Med Oncol 33: 49, 2016.

13. Gui F, Hong Z, You Z, Wu H and Zhang Y: MiR-21 inhibitor suppressed the progression of retinoblastoma via the modulation of PTEN/PI3K/AKT pathway. Cell Biol Int 40: 1294-1302, 2016.

14. Bai S, Tian B, Li A, Yao Q, Zhang G and Li F: MicroRNA-125b promotes tumor growth and suppresses apoptosis by targeting DRAM2 in retinoblastoma. Eye (Lond) 30: 1630-1638, 2016.

15. Zhang $\mathrm{Y}$, Xue C, Zhu X, Zhu X, Xian H and Huang Z: Suppression of microRNA-125a-5p upregulates the TAZ-EGFR signaling pathway and promotes retinoblastoma proliferation. Cell Signal 28: 850-860, 2016.

16. Zeng Q, Jin C, Chen W, Xia F, Wang Q, Fan F, Du J, Guo Y, Lin C, Yang K, et al: Downregulation of serum miR-17 and miR-106b levels in gastric cancer and benign gastric diseases. Chin J Cancer Res 26: 711-716, 2014.

17. Xu X, Liu Z, Wang J, Ling Q, Xie H, Guo H, Wei X, Zhou L and Zheng S: miRNA profiles in livers with different mass deficits after partial hepatectomy and miR-106b 25 cluster accelerating hepatocyte proliferation in rats. Sci Rep 6: 31267, 2016.

18. Yu D, Shin HS, Lee YS and Lee YC: miR-106b modulates cancer stem cell characteristics through TGF- $\beta /$ Smad signaling in CD44-positive gastric cancer cells. Lab Invest 94: 1370-1381, 2014.

19. Dai F, Liu T, Zheng S, Liu Q, Yang C, Zhou J, Chen Y, Sheyhidin I and Lu X: MiR-106b promotes migration and invasion through enhancing EMT via downregulation of Smad 7 in Kazakh's esophageal squamous cell carcinoma. Tumour Biol 37: 14595-14604, 2016.

20. Li N, Liu Y, Miao Y, Zhao L, Zhou H and Jia L: MicroRNA-106b targets FUT6 to promote cell migration, invasion, and proliferation in human breast cancer. IUBMB Life 68: 764-775, 2016.

21. Ishaq SM, Kehar SI, Zafar S and Hasan SF: Correlation of CD24 expression with histological grading and TNM staging of retinoblastoma. Pak J Med Sci 32: 160-164, 2016.

22. Livak KJ and Schmittgen TD: Analysis of relative gene expression data using real-time quantitative PCR and the 2(-Delta Delta C(T)) method. Methods 25: 402-408, 2001. 
23. Zhang R, Wang W, Li F, Zhang H and Liu J: MicroRNA-106b 25 expressions in tumor tissues and plasma of patients with gastric cancers. Med Oncol 31: 243, 2014.

24. Verboon LJ, Obulkasim A, de Rooij JD, Katsman-Kuipers JE, Sonneveld E, Baruchel A, Trka J, Reinhardt D, Pieters R, Cloos J, et al: MicroRNA-106b 25 cluster is upregulated in relapsed MLL-rearranged pediatric acute myeloid leukemia. Oncotarget 7: 48412-48422, 2016.

25. Zheng L, Zhang Y, Liu Y, Zhou M, Lu Y, Yuan L, Zhang C, Hong $\mathrm{M}$, Wang S and Li X: MiR-106b induces cell radioresistance via the PTEN/PI3K/AKT pathways and p21 in colorectal cancer. J Transl Med 13: 252, 2015.

26. Kim K, Chadalapaka G, Pathi SS, Jin UH, Lee JS, Park YY, Cho SG, Chintharlapalli S and Safe S: Induction of the transcriptional repressor ZBTB4 in prostate cancer cells by drug-induced targeting of microRNA-17-92/106b-25 clusters. Mol Cancer Ther 11: 1852-1862, 2012

27. Yang WS, Chadalapaka G, Cho SG, Lee SO, Jin UH, Jutooru I, Choi K, Leung YK, Ho SM, Safe S and Kim K: The transcriptional repressor ZBTB4 regulates EZH2 through a MicroRNA-ZBTB4-specificity protein signaling axis. Neoplasia 16: 1059-1069, 2014.
28. Kim K, Chadalapaka G, Lee SO, Yamada D, Sastre-Garau X, Defossez PA, Park YY, Lee JS and Safe S: Identification of oncogenic microRNA-17-92/ZBTB4/specificity protein axis in breast cancer. Oncogene 31: 1034-1044, 2012.

29. Yamada D, Pérez-Torrado R, Filion G, Caly M, Jammart B, Devignot V, Sasai N, Ravassard P, Mallet J, Sastre-Garau X, et al: The human protein kinase HIPK2 phosphorylates and downregulates the methyl-binding transcription factor ZBTB4. Oncogene 28: 2535-2544, 2009.

30. Guarnieri AL, Towers CG, Drasin DJ, Oliphant MUJ, Andrysik Z, Hotz TJ, Vartuli RL, Linklater ES, Pandey A, Khanal S, et al: The miR-106b-25 cluster mediates breast tumor initiation through activation of NOTCH1 via direct repression of NEDD4L. Oncogene, Apr 17, 2018 (Epub ahead of print).

31. Gruszka R and Zakrzewska M: The oncogenic relevance of miR-17-92 cluster and its paralogous miR-106b-25 and miR-106a-363 clusters in brain tumors. Int J Mol Sci 19: pii: $\mathrm{E} 879,2018$.

c) (i) $($ ) This work is licensed under a Creative Commons Attribution-NonCommercial-NoDerivatives 4.0 International (CC BY-NC-ND 4.0) License. 